\title{
Promoting healthy lifestyle habits through learning based on active video games
}

\author{
Nazaret Gómez del Río \\ Grupo Grial. Universidad de Salamanca \\ Salamanca, España. \\ ngomrio@usal.es
}

\author{
Carina S. González-González \\ Grupo ITED. Universidad de La Laguna \\ La Laguna, España \\ carina.gonzalez@ull.edu.es
}

\author{
Francisco J. García-Peñalvo \\ Grupo GRIAL. Universidad de Salamanca \\ Salamanca, España. \\ fgarcia@usal.es
}

\begin{abstract}
The overweight and obesity have been declared as a worldwide health problem. Active videogames and technologies can be used as attractive tools to support educational interventions with children. Thus, in this paper, we present an educational program to promote healthy habits in children with obesity using active videogames and motor play as main strategies. This program was developed with 46 children and their parents in collaboration with the hospital and schools. The results show positive effects in the knowledge about healthy habits and behaviors of children.
\end{abstract} habits

Keywords—active videogames, obesity, overweight, healthy

\section{INTRODUCTION}

Obesity has been progressively increasing in recent decades. It has become one of the most serious public health problems in the pediatric population, estimating that about 41 million children under five years of age were overweight or obese in 2016. If current trends continue, the number will increase to 70 million by 2025 [1]. Overweight and obesity are the fifth leading risk factor for death worldwide and are attributed to nearly three million deaths per year [2]. Health problems resulting from being overweight or obese are estimated to account for $58 \%$ of the burden of diabetes, $21 \%$ of ischemic heart disease, and between $8 \%$ and $42 \%$ of some cancers, and these risks grow in proportion to the increase in body weight and represent a significant burden on quality of life.

In this regard, technological advances have opened up a new range of possibilities with the emergence of active video games that allow interventions to be approached from a more attractive perspective for children [3]. Active video games cover different modalities, including the simulation of different sports activities, dance, and games based on the video capture of the body movement itself, becoming audiovisual and interactive support to the practice of exercise $[4,5]$.

Given the above, this paper presents the development of a study that assesses the effectiveness of an educational intervention model using active video games and motor games in changing attitudes and healthy habits in children with obesity, as well as serving as a tool for the prevention and promotion of health in healthy lifestyles from the family environment. The article is structured as follows: first, the PROVITAO project [6], its hypotheses, objectives, and method are presented. Then, the project's main results are presented, as well as the conclusions on the results obtained.

\section{Provitao}

\section{A. Hypothesis and objectives}

The proposed intervention is an effective tool to promote the acquisition of healthy habits in children and their families and impact improving the current and future quality of life of children suffering from childhood obesity.

The general objectives are as follows:

1. To promote the acquisition and permanence of healthy life habits in children with overweight/obesity through health education.

2. To evaluate the influence of the educational intervention program in overweight/obese children.

3. To promote social awareness of the importance of childhood obesity prevention (short and long-term complications, health, and social costs).

As for the specific objectives, we see the following:

1. To diagnose the situation of overweight/obese minors about their physical condition and needs.

2. To assess overweight children and their families' previous habits and lifestyles (habits, customs, and beliefs).

3. To give truthful information about healthy behavioral habits and to eliminate myths.

4. Create a family environment that reinforces the educational program on healthy eating habits and frequent physical activity.

5. Analyze the effectiveness of the intervention for the promotion of healthy habits through periodic evaluations.

6. Communicate and disseminate the activities and results obtained to the entire scientific, medical and social community.

7. To measure the child's motivation before the physical activity developed with motor games and active video games.

\section{B. Method}

This section describes the sample and working groups, the materials and methods used for the study, and the results obtained for each of the objectives proposed in this investigation. The study was approved by the Ethics Committee of the Hospital Universitario de Canarias (HUC) and by the University of La Laguna (ULL CEIBA 20200410), 
The study design is quasi-experimental, longitudinal, and prospective over three years. It consists of two main phases, and in each phase, there is a control group and an experimental group. The same intervention is repeated in each phase (Figure 1).
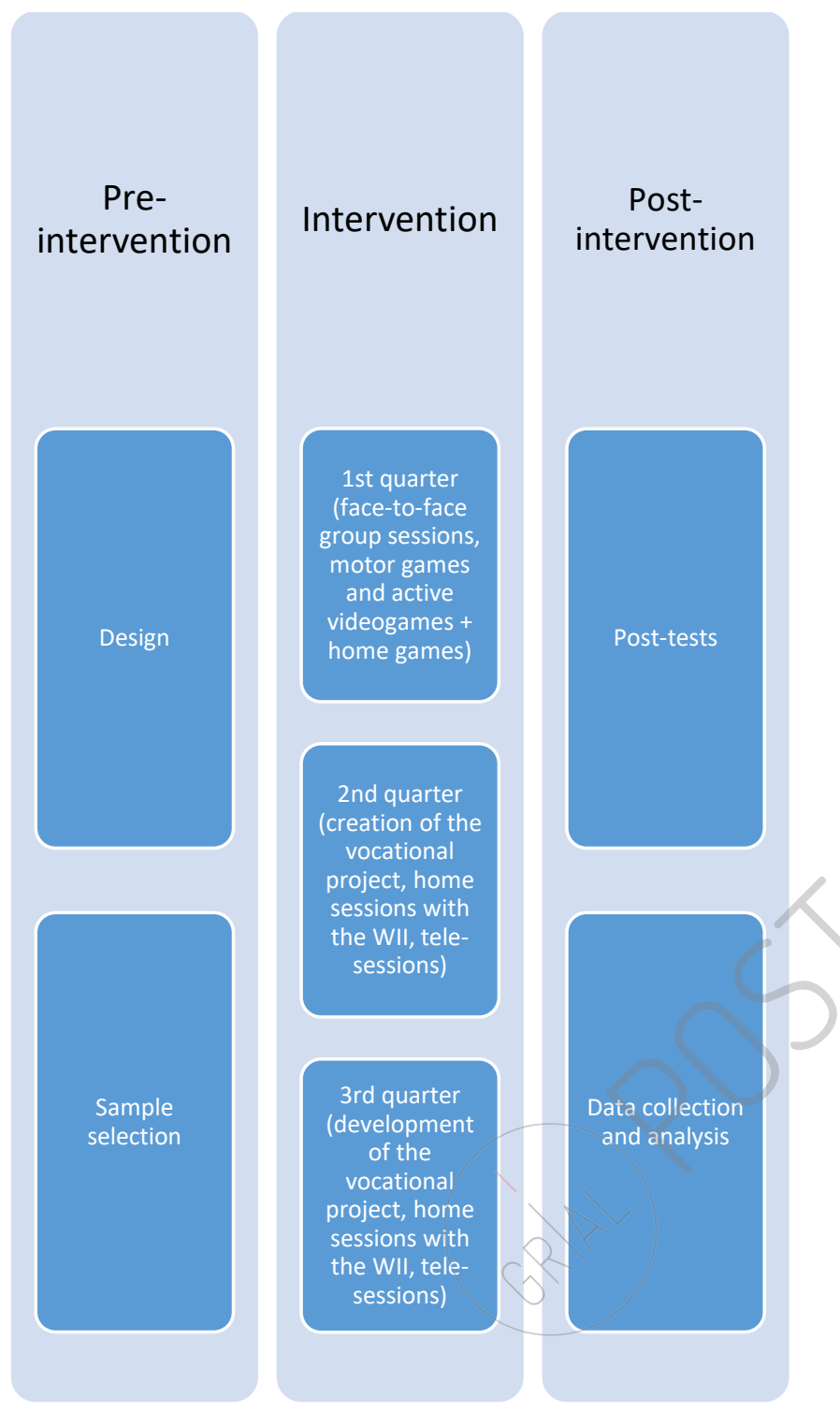

Figure 1. Structure of the intervention program

The sample consisted of a total of 46 boys and girls between [6-12] years of age with BMI above the 97th percentile (an indicator of childhood obesity). The sample is organized by phases, in Phase 1:

G1: Experimental Group (boys and girls with obesity participating in the intervention program) composed of 13 participants ( 8 girls and 5 boys).

G2: Control Group (boys and girls with obesity not participating in the intervention program) composed of 10 participants ( 3 girls and 7 boys).

In Phase 2:
G3: Experimental Group (boys and girls with obesity participating in the intervention program) composed of 12 participants ( 7 girls and 5 boys).

G4: Control Group (boys and girls with obesity not participating in the intervention program) composed of 11 participants ( 7 girls and 4 boys).

The intervention program was carried out in its first year at the HUC, located in the province of Santa Cruz de Tenerife, Autonomous Community of the Canary Islands, Spain. The target population was children diagnosed with obesity seen in the outpatient pediatrics department of the HUC. In the second year, given that not enough cases were found within the pediatrics service of the HUC that met the inclusion criteria, the sample was selected from the population of 6 public schools in the district of La Laguna, such as CEIP Aneja or CEIP Las Mercedes. The population was the students enrolled in these participating schools with obesity.

During the design phase of the study, the following data collection instruments were elaborated and/or adapted: initial parents' questionnaire, children's healthy lifestyle questionnaire, BASC, player profile, KIDMED, EMODIANA, EMODIANA APP, Perceived Effort Scale for the child population, Wii Registry, Follow-up Notebooks, Satisfaction Questionnaire for parents, Satisfaction Questionnaire for children, Health Report, Playability Evaluation, and Usability Heuristics. In addition, templates were created for the collection of anthropometric measurements. Blood tests were collected from the HUC as well as medical records.

The main results of the developed intervention program are presented below.

\section{RESULTS}

The responses were analyzed and organized into five indexes according to their content regarding the parents' questionnaires. In four of them, higher scores indicate better habits, better attitude or more knowledge, as appropriate: eating habits of the children; parents' knowledge about food; parents' attitude towards video games; and adherence to the Mediterranean diet of their children as measured by the KIDMED questionnaire. In the case of the index, healthy habits of their children and leisure time habits, higher scores indicate worse habits from the point of view of health promotion. According to the information provided by the parents, the two groups of participants presented similar profiles at the beginning of the study. They report an improvement in healthy habits during the intervention, but that eating habits are lost in the long term (perhaps due to forgetfulness or lack of continuous training or lack of motivation towards healthy behaviors), these changes could have been influenced by the holiday periods before long-term follow-up, which can alter daily routines. There was an improvement in the free time habits of the children in the experimental group compared to the control group, although this did not reach statistical significance.

Regarding the attitude towards video games of the parents, we found a better attitude of the experimental group compared to the control group during the intervention, probably one of the benefits of the activities developed during the intervention phase with the children. In this sense, we would have succeeded in transmitting to the parents that video games can be a playful tool that allows them to practice, educate and/or 
reinforce good habits. However, this improvement is lost in the long term in both groups, and at least in the experimental group, it can be justified in return to the purely recreational use of video games.

Regarding the habits reported by the minors, we analyzed the responses in the questionnaires on health-related habits, organized into four indices: physical activity performed daily, self-perception of their motor competence (MC), and their opinion on the usefulness of Physical Activity or Sport (PA or Sport), knowledge of healthy eating and, finally, their perception of their health and well-being. In all cases, higher scores indicate better habits, knowledge, and satisfaction.

The results do not show a statistically significant improvement between the control and experimental groups in behaviors related to physical activity, their $\mathrm{CM}$, or their opinion about the usefulness of AFoD and perceived health and well-being. This suggests that there has not been a significant increase in physical activity in their daily lives, which could be justified as we saw in developing their vocational project involving physical activity. During the search for resources, we found that in some participants' areas of residence, there were no accessible and affordable resources for children that were also attractive to them. In some cases, public facilities allowed activities to be carried out (parks, avenues with equipment for physical activity, playgrounds), but this does not mean that the children were motivated to engage in these activities. However, when we assessed learning about healthy eating, the experimental group did show a significant improvement over the long-term control. Therefore, having participated in the experimental group seems to benefit since the knowledge acquired by the experimental group after the face-to-face intervention is maintained in the long term.

Regarding anthropometric and analytical measurements, no significant differences were found between the control and experimental groups or between the different times. Both groups, control and experimental, decreased BMI after the intervention and increased at follow-up. Therefore, we cannot conclude that the decrease is a product of the intervention performed.

In the only case studied with obesifyand prediabetes type II, improvements in BMI, waist circumference, and \% body fat can be observed and are maintained over time.

In the case of behavioral assessment, the "Behavioral Assessment System for Children and Adolescents - BASC" was applied in its self-report modality. In terms of group means and medians, these were generally in the "normal" range for both clinical and adaptive scales. On the other hand, if we look at the large standard deviations and the maxima and minima in most of them, we can see that scores indicative of poor fit are represented. This is mainly due to scores in the risk ranges and/or clinical significance of several participants in both groups at different assessment times. Focusing on changes over study time, we find that in the control group, attitudes towards school improve as they vary throughout the study, which coincides with the development of the school year; despite this, the measures of central tendency do not identify an overall negative attitude towards school or its usefulness. There are no significant changes in any other indicator or scale in the control group. There are more changes in the experimental group. For example, the presence of unusual thoughts, behaviors, or thoughts decreases throughout the intervention. The experimental group develops a sense of responsibility and control over issues related to their lives. Social stress, i.e., negative feelings associated with social interactions, are modified throughout the evaluated phases, and although the central tendency is maintained, the averages are decreasing. Something similar occurs in the depression index, which decreases the variability of the scores with central tendencies.

As for the group intervention based on games, the results of the emotional analysis carried out on the participants at the entrance and exit of the face-to-face sessions give us an indicator of the motivation with which they attended the intervention. However, we are especially interested in the informative nature of the emotional impact of the intervention. Thus, to assess the emotional effect of gamification, the game dynamics carried out and the training. The children generally attended the sessions feeling positive emotions maintained at the end of the sessions, with little representation of negative or neutral emotions both at the beginning and at the end of the sessions. The intervention shows a tendency to favor positive emotions, and based on the justifications made; we see that these are related to the elements of the activity carried out (games, participants, companions, learning), in addition to making reference to personal motives, highlighting the verbalizations of having fun and having a good time. These results reaffirm the motivational value of face-to-face sessions, especially motor games and training games, which manage to establish positive expectations from one session to the next.

Concerning the physical activity developed during the face-to-face interventions through motor games, the effort perceived by the children was analyzed through the application of a self-report scale. At the end of the session, each participant individually indicated how the physical activity had gone, selecting the label that best defined him or her from a scale with 10 levels ranging from "Very, very easy" to "So hard that I have to stop." According to the information provided, the motor games performed were perceived as medium and/or low intensity, mostly $(88.9 \%)$ within easy to specific categories. However, the data collected by the sensors show that the activity performed in some cases was moderate to intense, reaching peaks close to $200 \mathrm{ppm}$ in some cases. Therefore, although the activities demanded a medium effort, it was an intensely physical activity for some children, and some expressed this in their perceptions $(8.2 \%$ a little effort and $3.4 \%$ higher intensity effort). The professional team also made this observation, indicating that some of the children showed evidence of being doing an "intense" physical activity and enjoyed the activity, and still considered that the activity had been easy (in some of its degrees). In addition to individual differences, which can vary the impact of the same activity for different participants, motivation and the adequate adjustment of the skills required for the performance of motor games can be determining factors so that the physical effort that may have been made does not negatively affect the perception of effectiveness and therefore of the ease of the physical activity performed.

\section{CONCLUSIONS}

Given the experience, we have developed and the literature reviewed, knowing the fundamental role of parents/guardians as caregivers and agents in learning and/or changing habits at early ages, given the fluctuations in eating habits that seem to be stationary as they affect both groups 
equally, we believe that future interventions should focus on parents in the early stages and highlight the importance of making good nutrition, physical activity and other healthy habits a lifestyle so that compliance does not depend on external variables and does not involve a continuous effort. Of particular importance in this regard are the active involvement of fathers and mothers in physical and/or sports activities as the main role models, promoters, and supervisors. It would also be advisable to have more activities offered by the public administration (schools, town councils, associations, etc.) designed for children that involve physical activity and do not necessarily sport since, as we have seen, physical activity in a recreational context is motivating and could be effective with children who do not show specific preferences for sports.

Concerning the emotional and behavioral changes described and which primarily affect the experimental group in the long term, they indicate that maintaining group cohesion through biweekly meetings and videoconferences, once the first phase of the intervention was over, favored the maintenance of the social and personal benefits of the intervention. Thus, these results support the usefulness of the intervention aimed at favoring the social relationships and safety of the participants, since, although as a population no clinically significant scores were detected, in the individual review, we can see that in this population there is a high prevalence of indicators of social and personal maladjustment. This is why the intervention carried out, and its effectiveness becomes essential, not only in the promotion of healthy lifestyle habits but also in the promotion of satisfactory social relations and the perception of control over one's own life, that is to say, favoring the integral health of the minors.

We believe that it would be interesting to integrate this type of program into the educational model of the centers since it is a program that educates and motivates behavioral change from an early age using the dynamics of games, which are so close to the children.

We also consider that it is necessary to introduce in the short term space for education in healthy lifestyle habits in the educational curriculum of minors. This type of education should not be left to chance and to the teacher's interest in training his or her students in this subject. Education in healthy lifestyle habits is part of the curriculum that would ensure the long-term acquisition of healthy behaviors and prevent non- communicable diseases related to bad habits, resulting in improved health for the whole society.

Finally, remember that the acquisition of healthy lifestyle habits involving reducing obesity to prevent additional metabolic alterations should be a priority for prevention in boys and girls.

\section{ACKNOWLEDGMENTS}

The authors of this article wish to thank the entire PROVITAO Project team and the participating children, schools, and families. This work has been funded by the CajaCanarias Foundation, PROVITAO Project, Reference OBE05.

\section{REFERENCES}

[1] Agencia Española de Seguridad Alimentaria. Estrategia NAOS. Estrategia para la nutrición, actividad física y prevención de la obesidad. Invertir la tendencia de la obesidad [en línea]. 2005 [fecha de acceso 15 de mayo de 2021]. Disponible en: https://www.aesan.gob.es/AECOSAN/docs/documentos/nutricion/estr ategianaos.pdf

[2] World Health Organization. El periodo de vida sana puede prolongarse entre cinco y diez años segun la OMS [en línea]. 2002 [fecha de acceso 15 de mayo de 2021]. Disponible en: http://www.who.int/mediacentre/news/releases/pr84/es/

[3] De Bourdeaudhuij I, Maes L, De Henauw S, De Vriendt T, Moreno LA, Kersting M, etal. Evaluation of a Computer-Tailored Physical Activity Intervention in Adolescents in Six European Countries: The Activ-O-Meter in the HELENA Intervention Study. J Adolesc Heal. mayo de 2010;46(5):458-66

[4] González-González, C.S.; Gómez del Río, N.G.; Toledo-Delgado, P.A.; García-Peñalvo, F. (2021). Active game-based solutions for the treatment of childhood obesity. Sensors 2021, 21(4), 1266 (JCR, Q1). doi: https://doi.org/10.3390/s21041266

5] del Val, D. D., \& Llorente, Á. M. (2019). Efectos de una intervención de ejercicio físico con videojuegos activos en la composición corporal de niños con sobrepeso y obesidad. Trabajo Fin de Grado. Universidad de Zaragoza

[6] Del Rio, N. G., González, C. S. G., González, R. M., Adelantado, V. N., Delgado, P. T., \& Fleitas, Y. B. (2018, April). Gamified educational programme for childhood obesity. In 2018 IEEE Global Engineering Education Conference (EDUCON) (pp. 1962-1668). IEEE.

[7] Del Río, N. G., González-González, C. S., Martín-González, R., Navarro-Adelantado, V., Toledo-Delgado, P., \& García-Peñalvo, F. (2019). Effects of a gamified educational program in the nutrition of children with obesity. Journal of medical systems, 43(7), 1-12. 